www.nature.com/ijo

\title{
CORRIGENDUM
}

\section{FAAH deficiency promotes energy storage and enhances the motivation for food}

\author{
C Touriño, F Oveisi, J Lockney, D Piomelli and R Maldonado
}

International Journal of Obesity (2012) 36, 477; doi:10.1038/ijo.2011.112

Correction to: International Journal of Obesity (2009) 34, 557-568; doi:10.1038/ijo.2009.262

Since the publication of the above paper, the authors have noticed that the affiliations of Dr D Piomelli were incorrectly listed. The correct details are as follows: Departments of
Pharmacology and Biological Chemistry, University of California, Irvine, Irvine, CA, USA and Unit of Drug Discovery and Development, Italian Institute of Technology, Genoa, Italy.

The authors would like to apologize for the error. 